R\$2,000.00/month (Minimum: 0, Maximum: 12,000). The prevalence for HIV was 23\% (41/180, 95\% CI: 16,87-29.61). In multiple regression analysis, the following variables were included in the model: history of STI (sexually transmitted infection) $(p=0.00)$; history of forced sexual intercourse ( $p$ $=0.01)$; sexual initiation before age $13(\mathrm{p}=0.08)$; use of crack $(p=0.02)$ and cocaine $(p=0.12)$; relationship with a drug using partner $(p=0.09)$, and prostitution $(p=0.13)$. According to the model, STI history (OR[odds ratio]: 4.4; 95\% CI: 1.99-9.70) and forced sexual intercourse (OR: 2.2; 95\% CI 1.01-4.27), were independently associated with HIV.

Conclusion It is essential to implement public policies that meet the specific needs of transgender and transvestite women, given their high infection rates and related risk factors.

Disclosure No significant relationships.

\section{P779 PRE-EXPOSURE PROPHYLAXIS USE AMONG TRANS AND GENDER DIVERSE PEOPLE: CHARACTERISTICS AND BEHAVIOUR FROM THE EPIC-NSW TRIAL}

\begin{abstract}
${ }^{1}$ Stefanie Vaccher, ${ }^{2}$ Rebecca Guy, ${ }^{3}$ David Templeton, ${ }^{4}$ Iryna Zablotska-Manos, ${ }^{2}$ Shawn Clackett, ${ }^{5}$ Martin Holt, ${ }^{6}$ Garrett Prestage, ${ }^{7}$ Janaki Amin, ${ }^{8}$ Christine Selvey, ${ }^{1}$ Fengyi Jin, ${ }^{2}$ Andrew Grulich, ${ }^{6}$ Benjamin Bavinton*. ${ }^{1}$ The Kirby Institute, UNSW Sydney, Sydney, Australia; ${ }^{2}$ Kirby Institute, Sydney, Australia; ${ }^{3}$ RPA Sexual Health, Sydney, Australia; ${ }^{4}$ Sydney Medical School Westmead, University of Sydney, Sydney, Australia; ${ }^{5}$ Centre for Social Research in Health, UNSW Sydney, Sydney, Australia; ${ }^{6}$ UNSW Sydney, The Kirby Institute, Sydney, Australia; ${ }^{7}$ Macquarie University, Sydney, Australia; ${ }^{8}$ NSW Ministry of Health, Sydney, Australia
\end{abstract}

\subsection{6/sextrans-2019-sti.837}

Background Trans and gender diverse (TGD) individuals are at higher risk of HIV than the general population. TGD individuals are under-represented among HIV pre-exposure prophylaxis (PrEP) users, hence little is known about their pill-taking behaviours. We describe demographic characteristics and PrEP adherence by TGD individuals in the EPIC-NSW study.

Methods From March 2016 to April 2018, 9,708 individuals were enrolled in EPIC-NSW. At baseline, then quarterly, participants were invited to complete an optional online behavioural and adherence survey. Factors previously associated with lower PrEP adherence were compared between TGD and nonTGD participants using chi-squared tests.

Results Of the 6,942 EPIC-NSW participants that completed any survey, 96 identified as TGD (1.4\%), including 38 trans women and 15 trans men. TGD participants were significantly younger than non-TGD individuals (mean 34 vs 39 years, $\mathrm{p}<0.001)$ and less likely to be university educated $(44 \%$ vs $60 \%, \mathrm{p}=0.002)$. TGD individuals were more likely to identify as Indigenous Australian $(11 \%$ vs $2 \%, \mathrm{p}<0.001)$, although no more likely to have been born in Australia $(p=0.634)$. 52\% of TGD individuals reported being paid for sex by a man in the previous three months, compared to $8 \%$ of non-TGD participants $(p<0.001)$. TGD participants were as likely as other participants to report using crystal methamphetamine $(p=0.666)$, party drugs $(p=0.572)$, or condomless sex in the previous three months $(\mathrm{p}=0.991)$. Taking at least four or seven PrEP pills in the previous week was reported in $87 \%$ and $91 \%(p=0.071)$ and $77 \%$ and $82 \%(p=0.06)$ of surveys completed by TGD and non-TGD individuals, respectively.

Conclusion Despite increased levels of HIV-risk and socio-economic disadvantage, TGD participants had comparable levels of adherence to non-TGD EPIC-NSW participants. Additional services for TGD including counselling and PrEP monitoring within a supportive environment may be warranted to ensure ongoing protection against HIV.

Disclosure No significant relationships.

\section{P781 PATTERNS OF HIV AND STI AMONG TRANSGENDER WOMEN IN EASTERN AND SOUTHERN U.S.: INTERIM BASELINE FINDINGS FROM THE LITE COHORT}

${ }^{1}$ Andrea Wirtz, ${ }^{2}$ Kenneth Mayer, ${ }^{3}$ Asa Radix ${ }^{4}$ Charlotte Gaydos, ${ }^{5}$ Tonia Poteat ${ }^{1}$ Erin Cooney, ${ }^{6}$ Christopher Cannon, ${ }^{7}$ Allan Rodriguez, ${ }^{8}$ Andrew Wawrzyniak,

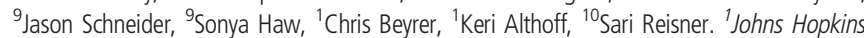
School of Public Health, Epidemiology, Baltimore, USA; ${ }^{2}$ Fenway Health/The Fenway Institute, Boston, USA; ${ }^{3}$ Callen-Lorde Community Health Center, New York, USA; ${ }^{4}$ Johns Hopkins University, Division of Infectious Diseases, Baltimore, USA; ${ }^{5}$ University of North Carolina School of Medicine, Center for Health Equity Research, Chapel Hill, USA; ${ }^{6}$ Whitman Walker Health, Washington, USA; ${ }^{7}$ University of Miami, School of Medicine, Miami, USA; ${ }^{8}$ University of Miami, Department of Psychiatry, Miami, USA; ${ }^{9}$ Emory University, School of Medicine, Atlanta, USA; ${ }^{10}$ Boston Children's Hospital, General Pediatrics, Boston, USA

10.1136/sextrans-2019-sti.838

Background Transgender women (TGW) in the U.S. experience a disproportionate burden of HIV and STIs. National HIV/STI surveillance does not report statistics for TGW. We describe the baseline prevalence and characteristics of HIV and STI among a cohort of TGW enrolled across 6 cities in eastern and southern US to-date.

Methods Adult TGW, regardless of HIV status, are recruited and enrolled in a baseline screening visit. Participants complete a socio-behavioral survey, oral HIV screening, urine and selfcollected anal and vaginal specimens for Neisseria gonorrhoeae (GC) and Chlamydia trachomatis (CT) testing, and serum treponemal syphilis testing and rapid plasma reagin (RPR) testing with quantitative RPR titers. Participants with negative HIV antibody test results at baseline and HIV-related risk behaviors are enrolled into the cohort.

Results Enrollment launched in March 2018. As of December 2018, 620 TGW completed baseline. Of these, 30\% were identified with HIV at baseline and 63\% enrolled into the HIV-uninfected cohort. Forty-percent reported a lifetime diagnosis of any STI. At baseline, 34\% of HIV-infected participants and $12 \%$ of HIV-uninfected participants had $\geq=1$ laboratory-confirmed STI. Baseline prevalence among all participants included: 2\% rectal GC, 0\% urogenital GC, 5\% rectal CT, $1 \%$ urogenital CT, and 13\% active syphilis determined by RPR and treponemal results. None of the 38 participants with self-reported history of vaginoplasty had GC or CT at the neovaginal site. Active syphilis infection was associated with residence in a southern city (aOR: 3.8, 95\%CI:1.7-8.6), identification as Black and/or Latinx (aOR: 3.7; 95\%CI:1.112.7), concern about safety in transit to healthcare (aOR: 1.9; 95\%CI:1.1-3.3), and positive baseline HIV antibody test (aOR: 3.1; 95\%CI:1.7-5.7).

Conclusion TGW in this 6-city baseline cohort have a high prevalence of HIV and STI. Study findings highlight demographic disparities, high HIV/syphilis co-infection, and barriers related to safety that may impede prompt diagnosis and treatment of STI and HIV in American TGW. Disclosure No significant relationships. 